\title{
The Phenomenology of Delirium: Presence, Severity, and Relationship between Symptoms
}

\author{
Soenke Boettger, ${ }^{1}$ Susanne Boettger, ${ }^{1}$ and William Breitbart ${ }^{2}$ \\ ${ }^{1}$ Department of Psychiatry and Psychotherapy, University Hospital Zurich, Rämistraße 100, 8091 Zurich, Switzerland \\ ${ }^{2}$ Department of Psychiatry and Behavioral Sciences, Memorial Sloan Kettering Cancer Center, 641 Lexington Avenue, \\ New York, NY 10022, USA
}

Correspondence should be addressed to Soenke Boettger; soenke.boettger@usz.ch

Received 31 July 2013; Revised 16 September 2013; Accepted 17 September 2013; Published 2 January 2014

Academic Editor: Shunichiro Shinagawa

Copyright (C) 2014 Soenke Boettger et al. This is an open access article distributed under the Creative Commons Attribution License, which permits unrestricted use, distribution, and reproduction in any medium, provided the original work is properly cited.

\begin{abstract}
Objective. To examine the phenomenological characteristics of delirium based on the Memorial Delirium Assessment Scale (MDAS) in order to explore the presence, severity of, and relationship between symptoms. Methods. An analysis of 100 cases of delirium recruited at Memorial Sloan Kettering Cancer Center (MSKCC) was performed. Sociodemographic and medical variables, the Memorial Delirium Assessment Scale (MDAS) subitems, and Karnofsky Performance Status scale (KPS) were analyzed of respect of the phenomenological characteristics and their interrelationship. Results. The most severe and frequent symptoms were recorded in the cognitive domain, psychomotor behavior, sleep-wake cycle, and disturbance of consciousness. Within the cognitive domain, concentration was the most severely affected task. The severity of impairment in most domains increased with delirium severity, whereas perceptual disturbances and delusions were independent of delirium severity. Advanced age and the prevalence of dementia increased with delirium severity in contrast to the functional status which declined. The presence of perceptual disturbances and delusions was independent of cognitive impairment and psychomotor abnormality, however, associated with the disturbances of consciousness and attention. Conclusion. Cognition, in particular concentration, was the most severely affected domain. Advanced age and the prevalence of dementia contributed to more severe delirium. Perceptual disturbances and delusions were independent of delirium severity; however, they were associated with disturbances of consciousness and attention.
\end{abstract}

\section{Introduction}

Delirium is a neuropsychiatric disorder which is characterized by disturbances of consciousness, attention, cognition, and perception with an abrupt onset and fluctuating course and an underlying physiological etiology [1]. Further frequent symptoms of delirium include various mood changes, sleepwake cycle disturbances, and psychomotor as well as language abnormalities [2].

The phenomenology of delirium has been examined in a number of studies. In a review [3], impairments in arousal have been recorded in $75 \%(48-82 \%)$, orientation in $76 \%$ $(62-100 \%)$, attention in $65 \%(17-100 \%)$, memory in $84 \%$ (64-100\%), thought process in $48 \%(2-68 \%)$, perception in $33 \%(20-78 \%)$, delusions in $27 \%(19-68 \%)$, psychomotor retardation in 59\% (53-60\%), psychomotor agitation in $44 \%$ (28-90\%), and sleep-wake cycle in 53\% (18-98\%).
The prevalence of perceptual disturbances and delusions was evaluated in further studies. Two studies revealed an approximately equal prevalence of perceptual disturbances and delusions (47.3 and 51.4\%, 32.6\% and $25.6 \%$, resp.) $[4,5]$. In contrast, another study documented a higher prevalence of perceptual disturbances [6].

Focusing on cognitive and noncognitive symptoms of delirium, inattention and sleep-wake cycle disturbance were recorded as most frequently impaired domains, whereas disorientation was recorded the least frequently domain. In this study, perceptual disturbances and delusions were not associated with cognitive deficits [7].

To date, evidence of the phenomenology of delirium remains conflicting and further studies are required in order to evaluate the phenomenology of delirium. One hundred cases of delirium were analyzed in respect of the MDAS 
subitems, and special attention was placed on the phenomenological characteristics of delirium, in particular the severity and presence of symptoms and their interrelationship, as well as differences in sociodemographic and medical variables over delirium severity.

\section{Methods}

2.1. Subjects. Subjects included patients referred for delirium management to the Memorial Sloan Kettering Cancer Center (MSKCC) Psychiatry Service from July to November 2000. MSKCC is a 452-bed private hospital specializing in the treatment of cancer, averaging more than 20,000 admissions every year. The Consultation-Liaison Psychiatry Service performs on average more than 2,000 consultations yearly.

Clinical data, including serial MDAS ratings, etiologies of delirium, medications and dosages utilized, adverse events, and comorbid medications as well as medical conditions, were recorded in a clinical database.

Inclusion criteria were meeting the DSM-IV-TR [8] criteria for delirium. Exclusion criteria were objections on the side of the patient or family to be evaluated for delirium, inability to comply with delirium rating, severe agitation, critical medical condition, and imminent death. All patients and families gave verbal consent to being evaluated for delirium. In patients with limited capacity to provide consent due to delirium, the patient's primary caregiver provided verbal consent to the patient's assent to treatment.

The data collection was performed by one physician dedicated to obtaining data and recorded in an IRB-approved MSKCC Psychiatry Service clinical database, and a waiver was obtained for the data analysis

2.2. Measurements. For the baseline assessment, the following information was collected: age, sex, cancer diagnosis, stage of cancer (localized, metastatic, or terminal), psychiatric diagnoses including a history of dementia, presence of brain metastases, and delirium etiologies.

Delirium severity was measured with the MDAS, a 10item, four-point clinician-rated scale (range of 0-30) which has been validated in cancer patients [9]. MDAS items range from 0 (absent) to 1 (mild), 2 (moderate), and 3 (severe) in presentation. The MDAS provides a clear description of the severity of presence of symptoms. Scale items assess disturbance in consciousness and level of consciousness as well as several areas of cognitive functioning, psychomotor activity, and sleep-wake cycle. An MDAS score of $>10$ identifies the presence of delirium $[10,11]$. The Karnofsky Performance Status scale (KPS) is a scale measuring functionality validated in cancer patients [12]. Scores of less than 50 indicate the inability to care for self, requiring hospital care, a score of 40 indicates a disability needing assistance, a score of 30 indicates a severe disability requiring hospital admission, and a score of 20 indicates needing hospital admission and active treatment representing a very sick patient.

Subtyping of delirium in the MDAS is based on the motor behavior $[2,13,14]$. Delirium severity is categorized by using the MDAS scores as follows: mild delirium $($ MDAS $<16)$, moderate delirium (MDAS 16-22), and severe delirium (MDAS > 22).

2.3. Statistical Analysis. Analyses were performed with the Statistical Package for the Social Sciences (SPSS) 20 statistical software package for Windows.

Descriptive statistics were used for the description of the sociodemographic, medical, and delirium variables. The primary interest were the phenomenology of delirium; mean scores and the presence of delirium in percent defined as moderate and severe symptomatology were determined. In order to evaluate mild, moderate, and severe delirium, the dataset was defined as a variable with three levels representing the severity of delirium (mild, moderate, and severe).

The data was parametric and nonparametric, contained interval, ordinal, and categorical variables. The independent samples $t$-test was computed in order to evaluate differences in age between mild, moderate, and severe delirium. The Kruskal-Wallis test was used for the comparison of three independent samples such as the delirium severity (mild, moderate, and severe) in respect of the severity of symptomatology based on the MDAS items. Pearson's chi-square $\left(\chi^{2}\right)$ was used for the comparison of categorical data such as the prevalence of cancer diagnoses between categories of delirium severity, as well as presence and interrelationship of delirium symptoms. For all implemented tests, post hoc, the alpha $(\alpha)$ was adjusted using the Bonferroni method. The significance level $\alpha$ was set at $P<0.05$.

\section{Results}

3.1. Sample Characteristics. The delirium sample represented an elderly population with diverse cancer diagnoses, a substantial rate of dementia, and brain metastases. The most frequent etiologies of delirium were the administration of opioids, infection, the administration of corticosteroids, and hypoxia.

Delirium severity as measured by MDAS was 19.17. Almost $70.6 \%$ of patients had moderate delirium, $16.7 \%$ had severe delirium, and $11.8 \%$ had mild delirium. Hypoactive delirium and hyperactive delirium were equally present. The level of functioning as measured with the KPS documented impairment (Table 1).

3.2. Phenomenology of Delirium. Based on mean MDAS scores, the most severe symptoms of delirium were impaired digit span, disorientation, psychomotor abnormalities, reduced ability to shift and maintain attention, sleep-wake cycle disturbances, thought disorder, and disturbance of consciousness in descending order. The least severe symptoms were delusions and perceptual disturbances (Table 2).

The prevalence of moderate and severe symptomatology across domains was different. Impaired digit span and psychomotor abnormality were the most common symptoms, followed by disorientation and reduced ability to shift attention, sleep-wake cycle disturbance, disorganized thinking, 
TABLE 1: Sociodemographic and medical variables of the delirium sample and severity of delirium.

\begin{tabular}{|c|c|c|c|c|c|}
\hline & $\begin{array}{l}\text { Delirium sample } \\
\quad(N=100)\end{array}$ & $\begin{array}{l}\text { Mild delirium } \\
\quad(N=12)\end{array}$ & $\begin{array}{l}\text { Moderate delirium } \\
\quad(N=71)\end{array}$ & $\begin{array}{l}\text { Severe delirium } \\
\quad(N=17)\end{array}$ & Statistical analysis \\
\hline Age & $58.4(19-89$, SD 16.7) & $51.9(19-78$, SD 18.8) & 57.7 (26-84, SD 15.9) & $66.4(30-89$, SD 16.6) & $t(27)=-2.19, P<0.05$ \\
\hline Gender in \% & & & & & ns \\
\hline Male & 51 & 33 & 54 & 53 & \\
\hline Female & 49 & 67 & 47 & 47 & \\
\hline Dementia in \% & 18 & 8.3 & 12.7 & 47.1 & $\chi^{2}=11.85(2), P<0.001$ \\
\hline Diagnoses in \% & & & & & ns \\
\hline Lung & 21 & - & 23.9 & 23.5 & \\
\hline Brain & 3 & - & 2.8 & 5.9 & \\
\hline Gastrointestinal & 14 & 8.3 & 14,1 & 17.6 & \\
\hline Sarcoma & 1 & 8,3 & - & - & \\
\hline Head and neck & 6 & 16.7 & 4.2 & 5.9 & \\
\hline Gynecological & 15 & 8.3 & 16.9 & 11.8 & \\
\hline Lymphoma & 13 & 41.7 & 8.5 & 11.8 & \\
\hline Other & 27 & 16.7 & 29.6 & 23.5 & \\
\hline Stage in $\%$ & & & & & ns \\
\hline Localized & 16.2 & 16.7 & 15.7 & 17.6 & \\
\hline Advanced & 78.8 & 83.3 & 81.4 & 64.7 & \\
\hline Terminal & 5.1 & - & 2.9 & 17.6 & \\
\hline Brain metastases in $\%$ & 24 & - & 25.4 & 35.3 & ns \\
\hline Etiologies in \% & & & & & ns \\
\hline Opioids & 63.4 & 63.6 & 62.7 & 66.7 & \\
\hline Corticosteroids & 30.1 & 27.3 & 32.8 & 20 & \\
\hline Infection & 40.9 & 63.6 & 38.8 & 33.3 & \\
\hline Hypoxia & 28 & 18.2 & 28.4 & 33.3 & \\
\hline CNS disease & 12.9 & - & 13.3 & 20 & \\
\hline Dehydration & 12.9 & 18.2 & 13.4 & 6.7 & \\
\hline Other & 12.9 & 9.1 & 11.9 & 20 & \\
\hline Type of delirium in \% & & & & & ns \\
\hline Hypoactive & 53 & 58.3 & 53.5 & 47.1 & \\
\hline Hyperactive & 47 & 41.7 & 46.5 & 52.9 & \\
\hline KPS & $35.5(20-50$, SD 7.70$)$ & $40(30-50$, SD 4.3$)$ & $35.8(20-50$, SD 7.3$)$ & $31.2(20-50$, SD 9.3) & $t(27)=3.06, P<0.01$ \\
\hline
\end{tabular}

KPS: Karnofsky Performance Status scale.

$\chi^{2}$ : Pearson's chi-square, SD: standard deviation.

and disturbance of consciousness. Perceptual disturbances and delusions were the least common symptoms.

3.3. Relationship between Symptoms. The presence of perceptual disturbances and delusions was independent of the cognitive domain and psychomotor abnormality; however, there was an association between disturbance of consciousness (perceptual disturbances: $\chi^{2}(1): 4.81, P<0.05$, and delusions: $\left.\chi^{2}(1): 9.52, P<0.01\right)$ and inability to shift and maintain attention (perceptual disturbances: $\chi^{2}(1): 6.41, P<$ 0.05 and delusions: $\left.\chi^{2}(1): 5.64, P<0.01\right)$ (Table 2 ).
3.4. Sample Characteristics over Severity of Delirium. With delirium severity, the age of patients and prevalence of dementia increased, and the level of functioning decreased. There were no differences in respect of gender distribution, cancer diagnoses, stage, presence of brain metastases, etiologies contributing to delirium, or type of delirium.

3.5. Symptomatology over Severity of Delirium. The symptomatology differed across mild, moderate, and severe delirium in severity (Table 3 ). In mild delirium, the psychomotor abnormality and the disturbance in sleep-wake cycle were the 
TABLE 2: Delirium phenomenology: prevalence of moderate and severe symptoms in \% and mean MDAS scores.

\begin{tabular}{llc}
\hline Delirium phenomenology & Mean MDAS scores & Presence in \% \\
\hline (1) Reduced consciousness/awareness & $1.89(1-3$, SD 0.62) & $75 \%$ \\
(2) Disorientation & $2.28(1-3$, SD 0.33) & $90 \%$ \\
(3) Short term memory impairment & $2.03(1-3$, SD 0.92) & $60 \%$ \\
(4) Impaired digit span & $2.45(1-3$, SD 0.58) & $96 \%$ \\
(5) Reduced ability to maintain/shift attention & $2.16(1-3$, SD 0.58) & $90 \%$ \\
(6) Disorganized thinking & $2.05(1-3$, SD 0.70) \\
(7) Perceptual disturbances & $1.08(0-3$, SD 1.08) & $78 \%$ \\
(8) Delusions & $0.92(0-3$, SD 0.90) & $34 \%$ \\
(9) Psychomotor abnormality & $2.22(1-3$, SD 0.50) \\
(10) Sleep-wake cycle disturbance & $2.08(1-3$, SD 0.56) & $28 \%$ \\
\hline
\end{tabular}

MDAS: Memorial Delirium Assessment Scale.

SD: standard deviation.

TABLE 3: Severity of symptomatology in mild, moderate, and severe delirium.

\begin{tabular}{lcccc}
\hline Delirium severity & Mild & Moderate & Severe & Kruskal-Wallis \\
\hline Reduced consciousness/awareness & $1.50(1-2$, SD 0.52) & $1.79(1-3$, SD 0.53) & $2.59($ SD 0.51) & $\chi^{2}(2): 26.86, P<0.001$ \\
Disorientation & $1.42(1-2$, SD 0.52) & $2.27(1-3$, SD 0.53) & $2.94(2-3$, SD 0.24) & $\chi^{2}(2): 38.66, P<0.001$ \\
Short term memory impairment & $1.00(1$, SD 0) & $2.00(1-3$, SD 0.89) & $2.88(2-3$, SD 0.33) & $\chi^{2}(2): 29.94, P<0.001$ \\
Impaired digit span & $1.75(1-2$, SD 0.45) & $2.44(1-3$, SD 0.53) & $3.00(3$, SD 0) & $\chi^{2}(2): 32.30, P<0.001$ \\
Reduced ability to maintain/shift attention & $1.67(1-2$, SD 0.49) & $2.07(1-3$, SD 0.49) & $2.88(2-3$, SD 0.33) & $\chi^{2}(2): 38.45, P<0.001$ \\
Disorganized thinking & $1.67(0-3$, SD 0.49) & $1.93(1-3$, SD 0.66) & $2.82(2-3$, SD 0.39) & $\chi^{2}(2): 26.97, P<0.001$ \\
Perceptual disturbances & $1.25(0-2$, SD 0.97) & $1.06(0-3$, SD 1.12) & $1.06(0-3$, SD 1.03) & ns \\
Delusions & $0.58(0-2$, SD 0.67) & $0.89(0-3$, SD 0.87) & $1.29(0-3$, SD 1.05) & $\mathrm{ns}$ \\
Psychomotor abnormality & $2.00(1-2$, SD 0.42) & $2.15(1-3$, SD 0.47) & $2.65(2-3$, SD 0.49) & $\chi^{2}(2): 16.24, P<0.001$ \\
Sleep-wake cycle disturbance & $2.00(1-2$, SD 0.60) & $2.01(1-3$, SD 0.52) & $2.41(1-3$, SD 0.61) & $\chi^{2}(2): 7.41, P<0.05$ \\
\hline
\end{tabular}

$\chi^{2}$ : Pearson's chi-square, SD: standard deviation.

most impaired domains followed by the cognitive domain and disturbance in consciousness. Perceptual disturbances and delusions were the least impaired domains. In moderate and severe delirium, the impairment within the cognitive domain was more severe followed by psychomotor abnormality, sleep-wake cycle, and disturbance in consciousness. In both moderate and severe delirium, the severity of perceptual disturbances and delusions was similar. Compared to the impairment in the cognitive domain and the disturbance in consciousness and sleep-wake cycle, the severity of perceptual disturbances and delusions appeared to be more subtle throughout mild, moderate, and severe delirium. Moreover, the severity of perceptual disturbances and delusions did not change between mild, moderate, and severe delirium (Kruskal-Wallis: ns) suggesting that the severity of perceptual disturbances and delusions may be independent of delirium severity. In contrast, the severity of impairment in the cognitive domain, disturbance of consciousness and sleepwake cycle, and psychomotor abnormality increased with delirium severity (Table 3 ).

\section{Discussion}

In this set of 100 cases, delirium was confirmed to represent a disorder of consciousness disturbance, cognition, and sleepwake cycle in which the impairment in the cognitive domain was the most prominent feature. In fact, concentration was the most affected cognitive task. Of interest, the severity of perceptual disturbances and delusions was independent of delirium severity. Advanced age and increased prevalence of dementia contributed to delirium severity, whereas the level of functioning declined with delirium severity. The presence of perceptual disturbances and delusions was not associated with cognitive impairment and psychomotor abnormality, however, associated with disturbance of consciousness and impairment in attention.

These findings regarding the prevalence of arousal disturbance, disorientation, cognitive impairment, psychomotor behavior abnormality, and sleep-wake cycle disturbance were compared in part to previous findings in respect of moderate and severe symptomatology $[3,4]$ and showed higher prevalence rates in respect of perceptual disturbances and delusions [5].

Impairment in the cognitive domain was the most prominent feature in this sample, and impaired concentration was the most severely affected task which confirmed previous findings [7]. Furthermore, disturbances of sleep-wake cycle and consciousness were also prominent features of delirium. In contrast, disorientation was more commonly present in this delirium sample, which could in part be due to the characteristics of the sample and the fact that the sample 
scored overall high on delirium severity, as disorientation appeared to increase over delirium severity.

A novel finding was that age and preexisting dementia contributed to delirium severity. The age of patients was higher, and the prevalence of delirium reached $50 \%$ in severe delirium. On the other hand, the functional status as measured with the KPS decreased over delirium severity indicating that more severe delirium was more disabling for the elderly patient with dementia. Furthermore, these patients have been shown to be at higher risk for developing delirium faced with more severe consequences, including higher risks for developing dementia [15, 16], persisting symptoms of delirium, and worse outcome in the months following an episode of delirium [17-19], as well as higher rates of institutionalization [20]. Dementia has been shown to have a compounding effect on delirium [21]. Thus, in part, higher delirium scores in patients with dementia were to be expected and could not be excluded.

Interestingly, the severity of perceptual disturbances and delusions was independent of delirium severity. Perceptual disturbances and delusions were documented in mild, moderate, and severe delirium, and the severity was not different, thus supporting findings that perceptual disturbances and delusions are independent of cognitive impairment [7]. The disturbance in consciousness, cognition, psychomotor behavior, and sleep-wake cycle increased with delirium severity. In respect of the presence of perceptual disturbances and delusions, psychomotor abnormalities were not associated with either, whereas the disturbance of consciousness and the inability to maintain and shift attention were.

Certain limitations have to be noted. The design of the study was cross-sectional; the evolution of symptoms over delirium severity cannot be reproduced. Longitudinal studies have suggested psychomotor abnormalities and sleepwake cycle disturbances in the early course of delirium [22] and disorientation, inattention, impaired memory, and sleep disturbances as more persistent symptoms throughout the course of delirium $[19,23]$. Within the sample of this study, patients with cognitive deficits were not excluded. Eighteen percent of patients had dementia and $24 \%$ had brain metastases. The effect on the outcome was negligible; an exclusion of patients with cognitive deficits did not alter the results. Furthermore, delirium phenomenology has not been shown to be altered by the presence of dementia [21]. The etiology of delirium in this sample was multifactorial and included medications with psychotropic effect. Of note, all subjects had cancer diagnoses and the generalizability of these results to the noncancer population may be limited. Further studies are required to understand the impact of etiological factors on the presentation of delirium and to characterize the phenomenology of delirium better.

In summary, delirium was confirmed to be a disorder of consciousness, cognition, and sleep-wake cycle. Cognitive impairment was the most prominent feature and increased with delirium severity. Within the cognitive domain, concentration was the most severely affected task. Further, the severity of perceptual disturbances and delusions was independent of delirium severity. In contrast, advanced age and dementia contributed to delirium severity. The presence of perceptual disturbances and delusions was not associated with cognitive impairment and psychomotor abnormality but with the disturbance of consciousness and attention.

\section{Conflict of Interests}

The authors declare that there is no conflict of intersts.

\section{References}

[1] W. Breitbart, J. Franklin, J. Levenson, D. R. Martini, and P. Wang, "Practice guideline for the treatment of patients with delirium," American Journal of Psychiatry, vol. 156, no. 5, supplement 1, pp. 1-20, 1999.

[2] D. J. Meagher, D. O'Hanlon, E. O’Mahony, P. R. Casey, and P. T. Trzepacz, "Relationship between symptoms and motoric subtype of delirium," Journal of Neuropsychiatry and Clinical Neurosciences, vol. 12, no. 1, pp. 51-56, 2000.

[3] S. B. Turkeil, P. T. Trzepacz, and J. C. Tavaré, "Comparing symptoms of delirium in adults and children," Psychosomatics, vol. 47, no. 4, pp. 320-324, 2006.

[4] J. Cutting, "The phenomenology of acute organic psychosis. Comparison with acute schizophrenia," British Journal of Psychiatry, vol. 151, pp. 324-332, 1987.

[5] R. Webster and S. Holroyd, "Prevalence of psychotic symptoms in delirium," Psychosomatics, vol. 41, no. 6, pp. 519-522, 2000.

[6] F. Sirois, "Delirium: 100 cases," Canadian Journal of Psychiatry, vol. 33, no. 5, pp. 375-378, 1988.

[7] D. J. Meagher, M. Moran, B. Raju et al., "Phenomenology of delirium: assessment of 100 adult cases using standardised measures," British Journal of Psychiatry, vol. 190, pp. 135-141, 2007.

[8] American Psychiatric Association, Diagnostic and Statistical Manual of Mental Disorders, American Psychiatric Association, Washington, DC, USA, 4th edition, 2000.

[9] W. Breitbart, B. Rosenfeld, A. Roth, M. J. Smith, K. Cohen, and S. Passik, "The memorial delirium assessment scale," Journal of Pain and Symptom Management, vol. 13, no. 3, pp. 128-137, 1997.

[10] J. Kazmierski, M. Kowman, M. Banach et al., "Clinical utility and use of DSM-IV and ICD-10 criteria and the memorial delirium assessment scale in establishing a diagnosis of delirium after cardiac surgery," Psychosomatics, vol. 49, no. 1, pp. 73-76, 2008.

[11] P. G. Lawlor, C. Nekolaichuk, and B. Gagnon, "Clinical utility, factor analysis, and further validation of the memorial delirium assessment scale in patients with advanced cancer: assessing delirium in advanced cancer," Cancer, vol. 88, no. 12, pp. 28592867,2000

[12] D. A. Karnofsky and J. H. Burchenal, "The clinical evaluation of chemotherapeutic agents in cancer," in Evaluation of Chemotherapeutic Agents, C. M. Macleod, Ed., pp. 191-205, Columbia University Press, New York, NY, USA, 1949.

[13] D. Meagher, M. Moran, B. Raju et al., "A new data-based motor subtype schema for delirium," Journal of Neuropsychiatry and Clinical Neurosciences, vol. 20, no. 2, pp. 185-193, 2008.

[14] D. J. Meagher, M. Moran, B. Raju et al., "Motor symptoms in 100 patients with delirium versus control subjects: comparison of subtyping methods," Psychosomatics, vol. 49, no. 4, pp. 300$308,2008$. 
[15] T. G. Fong, R. N. Jones, P. Shi et al., "Delirium accelerates cognitive decline in alzheimer disease," Neurology, vol. 72, no. 18, pp. 1570-1575, 2009.

[16] T. Rahkonen, H. Mäkelä, S. Paanila, P. Halonen, J. Sivenius, and R. Sulkava, "Delirium in elderly people without severe predisposing disorders: etiology and 1-year prognosis after discharge," International Psychogeriatrics, vol. 12, no. 4, pp. 473481, 2000.

[17] D. K. Kiely, R. N. Jones, M. A. Bergmann, K. M. Murphy, E. J. Orav, and E. R. Marcantonio, "Association between delirium resolution and functional recovery among newly admitted postacute facility patients," Journals of Gerontology A, vol. 61, no. 2, pp. 204-208, 2006.

[18] J. McCusker, M. Cole, N. Dendukuri, É. Belzile, and F. Primeau, "Delirium in older medical inpatients and subsequent cognitive and functional status: a prospective study," Canadian Medical Association Journal, vol. 165, no. 5, pp. 575-583, 2001.

[19] J. McCusker, M. Cole, N. Dendukuri, L. Han, and É. Belzile, "The course of delirium in older medical inpatients: a prospective study," Journal of General Internal Medicine, vol. 18, no. 9, pp. 696-704, 2003.

[20] S. K. Inouye, "Delirium in hospitalized older patients," Clinics in Geriatric Medicine, vol. 14, no. 4, pp. 745-764, 1998.

[21] P. T. Trzepacz, B. H. Mulsant, M. A. Dew, R. Pasternak, R. A. Sweet, and G. S. Zubenko, "Is delirium different when it occurs in dementia? A study using the delirium rating scale," Journal of Neuropsychiatry and Clinical Neurosciences, vol. 10, no. 2, pp. 199-204, 1998.

[22] J. R. Fann, C. M. Alfano, B. E. Burington, S. Roth-Roemer, W. J. Katon, and K. L. Syrjala, "Clinical presentation of delirium in patients undergoing hematopoietic stem cell transplantation: delirium and distress symptoms and time course," Cancer, vol. 103, no. 4, pp. 810-820, 2005.

[23] S. E. Levkoff, B. Liptzin, D. A. Evans et al., "Progression and resolution of delirium in elderly patients hospitalized for acute care," American Journal of Geriatric Psychiatry, vol. 2, no. 3, pp. 230-238, 1994. 


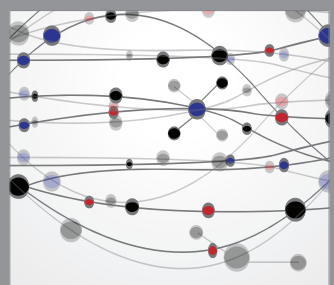

The Scientific World Journal
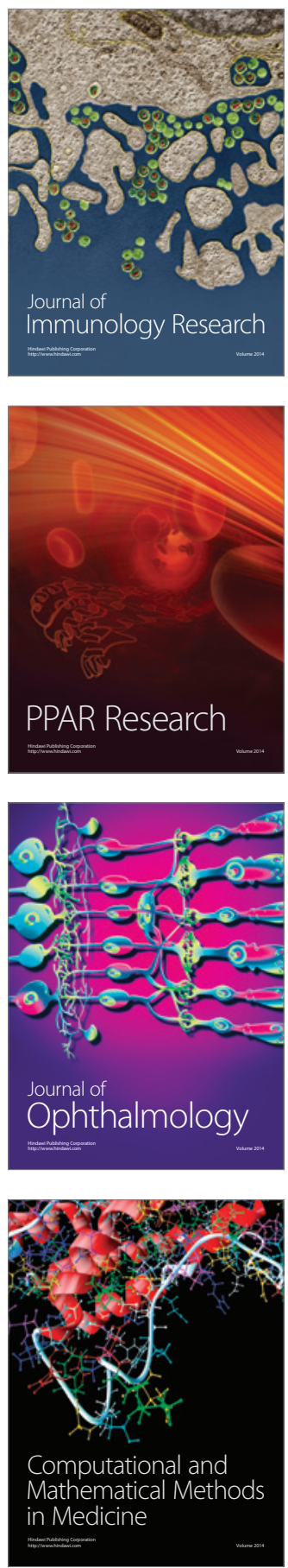

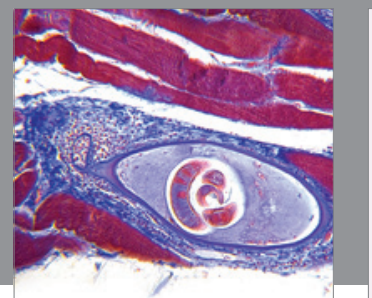

Gastroenterology

Research and Practice
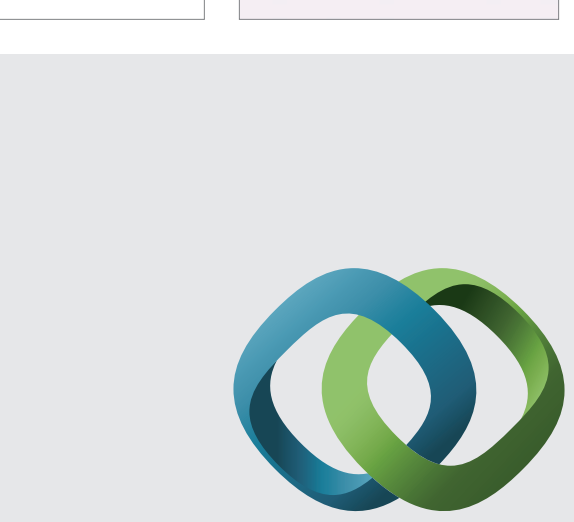

\section{Hindawi}

Submit your manuscripts at

http://www.hindawi.com
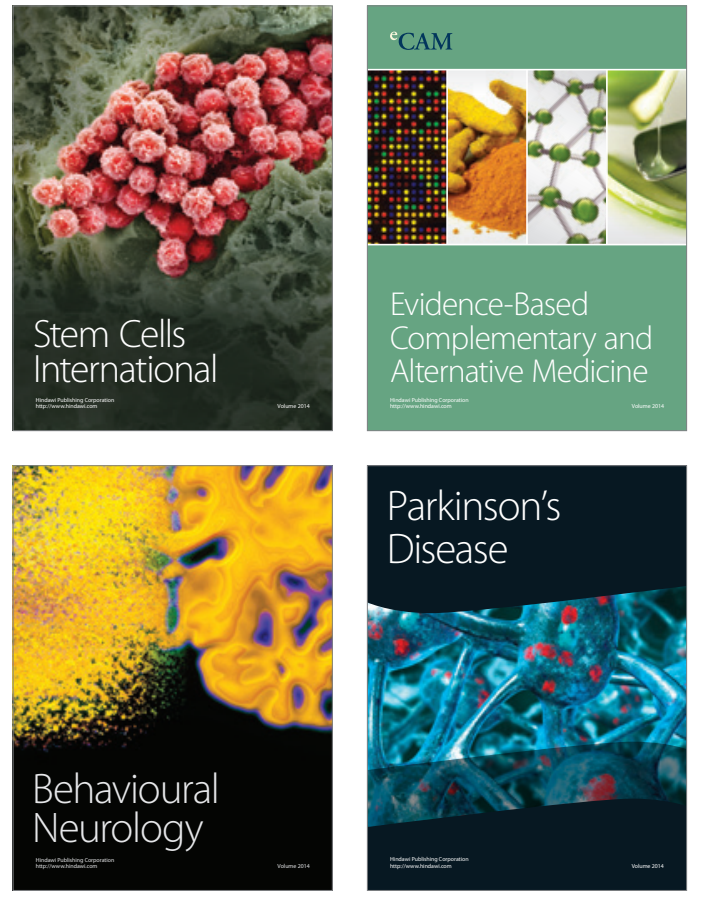
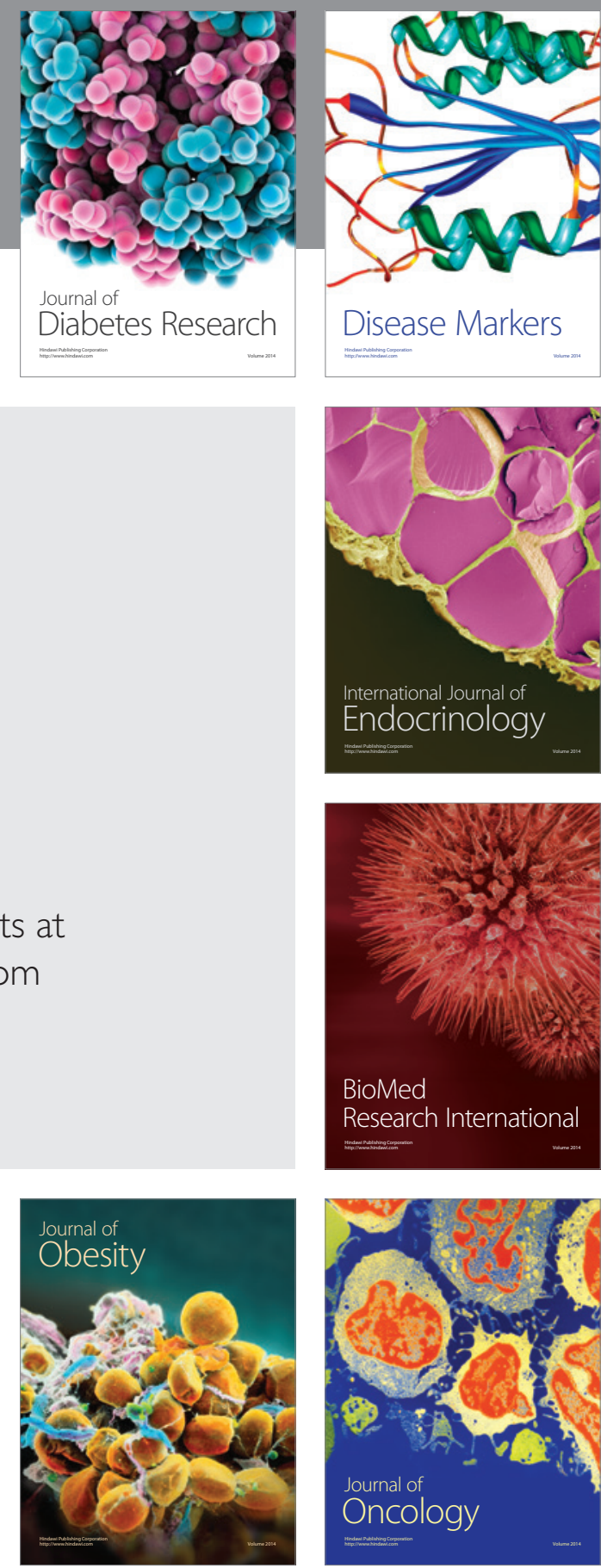

Disease Markers
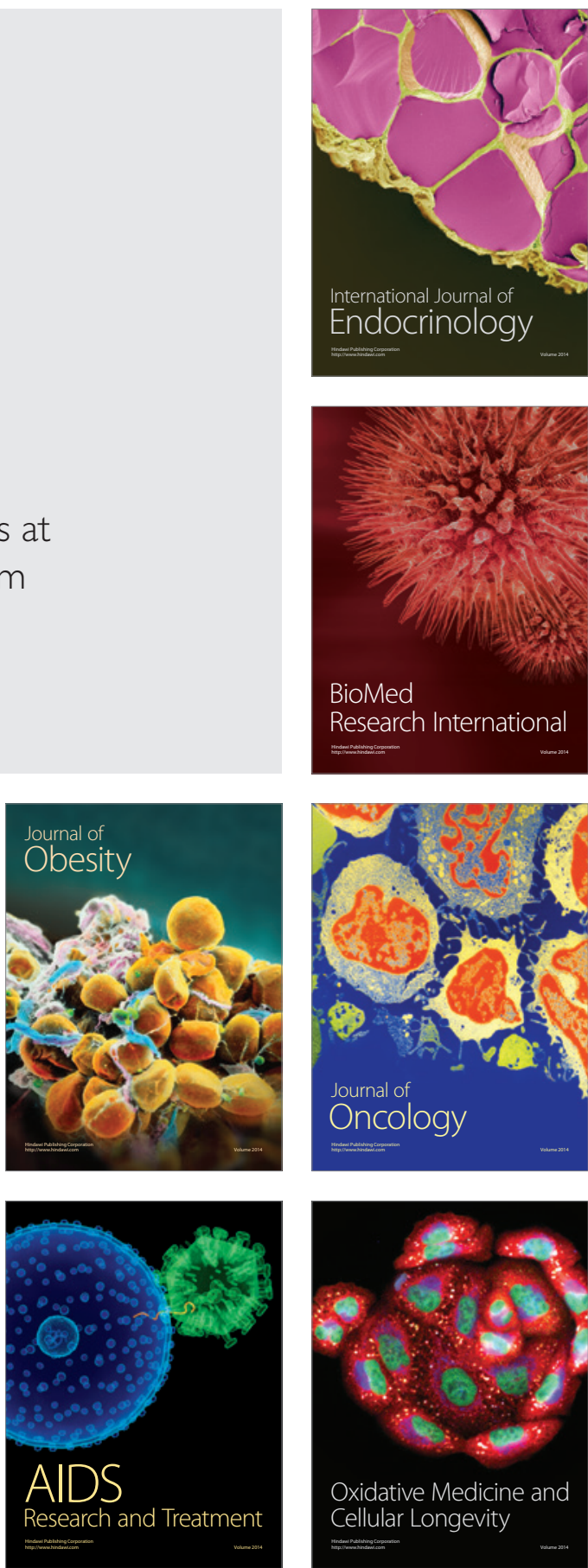